\title{
Literatur kompakt
}

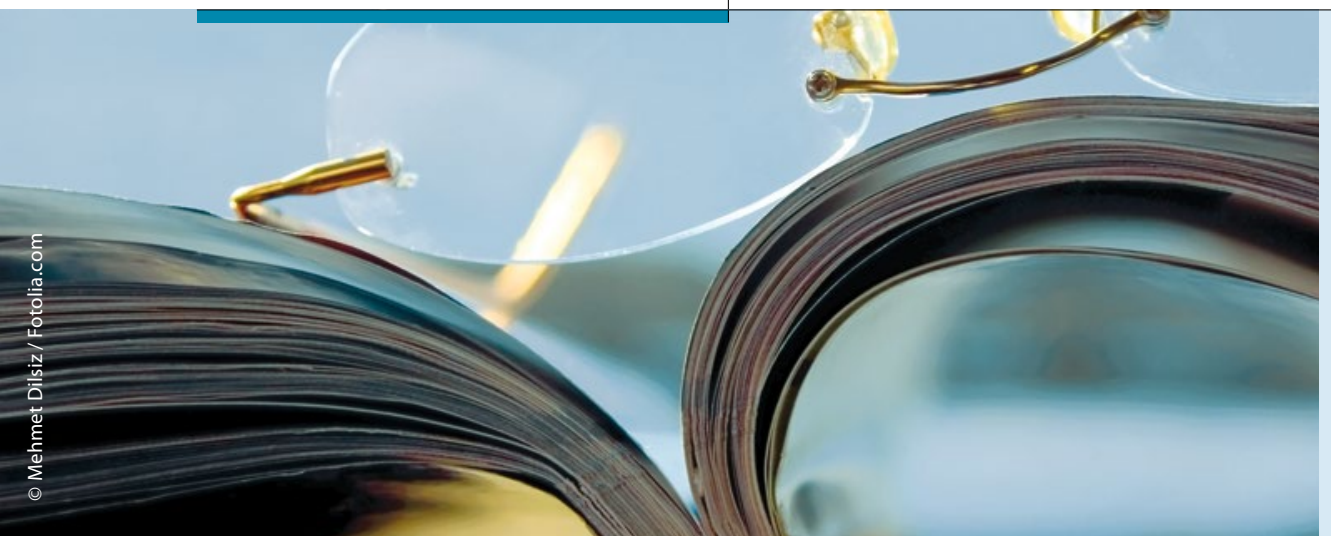

In der Rubrik "Literatur kompakt" werden die wichtigsten Originalarbeiten aus der internationalen Fachliteratur referiert.

\section{Nahrungsmittelallergien: Frühe Allergenkonfrontation kann schützen}

\section{Es mehren sich Hinweise, dass die frühe Einführung von potenziell allergenen Nahrungsmitteln bei gestillten Säuglingen vor Nahrungsmittelallergien schützt. Nun liegt eine randomisierte und kontrollierte Studie vor.}

\begin{abstract}
A ltere Leitlinien zur Prävention von Nahrungsmittelallergien empfehlen noch, allergene Nahrungsmittel möglichst spät in den Speiseplan von Säuglingen und Kleinkindern aufzunehmen. Dieser Ratschlag wurde ersatzlos gestrichen, nachdem sich in Beobachtungsstudien ein Vorteil für die frühe Zufütte-
\end{abstract}

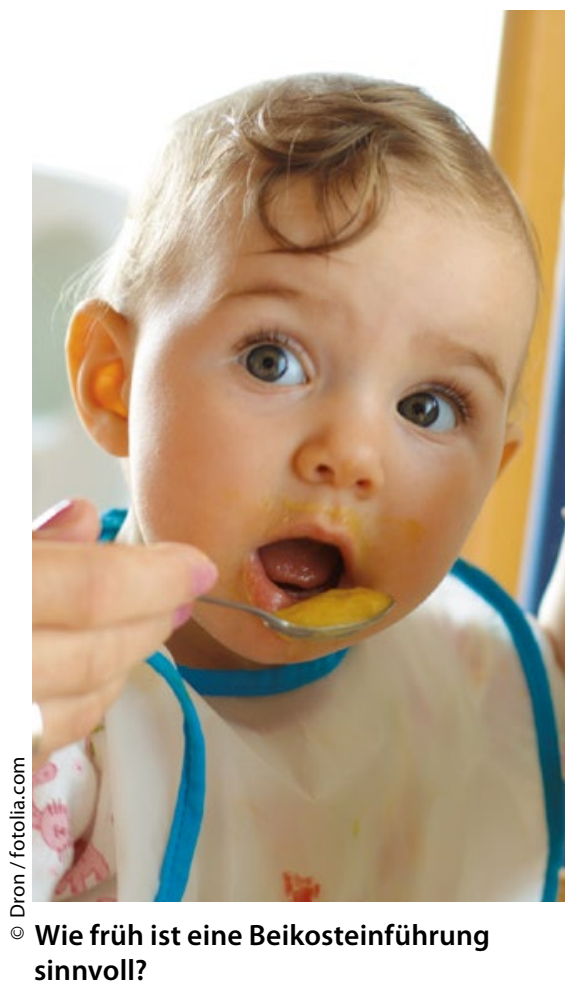

rung von Erdnüssen, Eiern, Kuhmilch oder Weizenprodukten ergeben hatte. In einer kontrollierten Studie mit Hochrisikokindern, die ein schweres Ekzem und/oder eine Eiallergie hatten, reduzierte der frühe Verzehr von Erdnüssen die Rate von Erdnussallergien im Alter von fünf Jahren um $80 \%$. Auch in nachfolgenden Untersuchungen blieb diese Toleranz erhalten. Daraufhin wurde die Studie "Enquiring about Tolerance“ (EAT) aufgelegt, in der mögliche protektive Effekte einer Früheinführung von verschiedenen allergenen Nahrungsmitteln bei Säuglingen ohne besonderes Allergierisiko untersucht werden sollte.

Einbezogen in die bevölkerungsbasierte Studie waren 1.303 bis dahin ausschließlich gestillte Säuglinge im Alter von drei Monaten, die nach AllergenHauttestung randomisiert entweder schon nach drei Monaten oder entsprechend den Standardempfehlungen nach sechs Monaten jeweils zweimal wöchentlich $2 \mathrm{~g}$ Allergenprotein unter anderem aus Erdnüssen, gekochtem Hühnerei, Kuhmilch und Weizen zusätzlich zu der normalen Beikost erhielten - vorausgesetzt, der Hauttest auf das Allergen war negativ. Nahrungsmittelallergien gegen eines oder mehrere der allergenen Nahrungsmittel im Alter zwischen einem und drei Jahren dienten als primäres Studienziel.
Im Rahmen der Intention-to-treatAnlyse entwickelten 7,1\% der Kinder in der Standardeinführungsgruppe und $5,6 \%$ in der Früheinführungsgruppe eine Nahrungsmittelallergie gegen mindestens eines der untersuchten Zielallergene, der Unterschied war mit $\mathrm{p}=0,32$ nicht signifikant, das Studienziel wurde nicht erreicht. Anders in der Per-Protokoll-Analyse: Hier war die Prävalenz von Nahrungsmittelallergien in der Früheinführungsgruppe mit $2,4 \%$ vs. $7,3 \%$ in der Standardgruppe signifikant niedriger $(\mathrm{p}=0,01)$.

Insgesamt, so die Autoren, war die frühe Einführung der Nahrungsmittelallergene zwar sicher, aber nicht einfach durchzusetzen, die Adhärenz in dieser Gruppe war dementsprechend schlecht. Doch zeigte sich bei der Analyse der wenig adhärenten vs. der adhärenten Teilnehmer, dass ein protektiver Effekt durch die allergenhaltigen Proteine dosisabhängig zu sein scheint. Zumindest bei Erdnüssen und Eiern schützt eine Dosis von mehr als $2 \mathrm{~g}$ pro Woche vor entsprechenden Nahrungsmittelallergien.

Fazit: In einer kontrollierten Studie zur Früheinführung von allergenen Nahrungsmitteln schon in Monat 3 ergab sich in der Intention-to-treat-Analyse kein Unterschied im Vergleich zum Standardvorgehen. Sekundäre Analysen lieferten jedoch Hinweise auf dosisabhängige Vorteile einer frühen „Desensibilisierung“.

Dr. Barbara Kreutzkamp

Perkin MR et al. Randomized trial of introduction of allergenic foods in breast-fed infants. New Engl J Med 2016;374:1733-43 